\title{
Extended scope physiotherapy roles for orthopedic outpatients: an update systematic review of the literature
}

This article was published in the following Dove Press journal:

Journal of Multidisciplinary Healthcare

4 February 2012

Number of times this article has been viewed

\author{
Jessica Stanhope' \\ Karen Grimmer-Somers' \\ Steve Milanese ${ }^{1,2}$ \\ Saravana Kumar' \\ Joanne Morris ${ }^{3}$ \\ International Centre for Allied \\ Health Evidence (iCAHE), University \\ of South Australia, Adelaide, Australia; \\ ${ }^{2}$ James Cook University, Townsville, \\ Australia; ${ }^{3}$ ACT Government Health \\ Directorate, Canberra, Australia
}

Correspondence: Karen Grimmer-Somers International Centre for Allied Health Evidence, University of South Australia, GPO Box 247I, Adelaide, Australia

Tel +6I 883022769

Fax +6I 883022853

Email karen.grimmer-somers@unisa.edu.au
Purpose: This systematic review updates one conducted in 2008 into extended scope practice (ESP) in physiotherapy in orthopedics.

Methods: A comprehensive open-ended search was conducted using electronic library databases and Google Scholar to identify any primary study design reporting on physiotherapists working in ESP roles within orthopedic settings. Studies were allocated to the National Health and Medical Research Council hierarchy of evidence, although only studies in levels I, II, or III_1 were critically appraised using a purpose-built critical appraisal tool. Information was extracted on the country of origin, ESP tasks, relevant training, patient types, health, process, and cost measures.

Results: 1071 studies were identified, and twelve were included in the review (including diagnostic and evaluative research). The hierarchy of evidence ranged from II to IV, from which only two diagnostic studies met the criteria for critical appraisal. ESP tasks included injection therapy, removing k-wires, and requesting investigations. The education of ESP physiotherapists varied widely, and included formal and informal training. The positive outcomes of ESP initiatives were reported, in diagnostic ability, reduced costs and waiting times, and improved health outcomes. Conclusion: Despite the positive results, the generally low level of evidence and the range of outcome measures reported, constrained clear conclusions regarding the health, process, and cost implications of ESP physiotherapy roles in orthopedic settings. The need for formalized, widely recognized training was highlighted, to give ESP physiotherapy roles credibility.

Keywords: extended scope practice, physiotherapy, orthopedics

\section{Introduction}

There are increasing reports, particularly from the United Kingdom (UK) and Australia, of implementation of extended scope of physiotherapy roles. A commissioned systematic review ${ }^{1}$ of the literature was undertaken to assist in determining the feasibility of introducing extended practice roles in physiotherapy within the Australian Capital Territory (ACT) Government Health Directorate and the ACT Department of Disability, Housing and Community Services (DHCS). This review found a lack of consensus regarding the definition of extended scope practice (ESP), although there was general agreement that it involved an expansion of traditional physiotherapeutic roles, in terms of diagnostics, management, and consultation. This review presented a working definition of ESP in physiotherapy:

"An ESP physiotherapist is a clinical specialist, who has the opportunity to develop and demonstrate expertise beyond the currently recognized scope of practice, including some aspect of job enhancement or expansion, involving the areas of extended therapeutics, diagnostics and practice consultation." 
Lowe et $\mathrm{al}^{1}$ incorporated peer-reviewed and non-peerreviewed publications, and grey literature. This review identified that while the majority of literature supported the potential usefulness of extending traditional roles in physiotherapy, there was a lack of information on quantifiable outcomes, particularly in terms of clinical efficacy, safety, and cost reductions or benefits. The literature presented in the 2008 review focused on organizational issues, such as patient waiting times, re-referral rates, and the appropriateness of referrals made by the ESP physiotherapist.

Lowe et al's' review examined ESP physiotherapy roles within emergency departments (EDs) and orthopedic outpatient clinics, although the potential for ESP in cardiorespiratory, obstetrics and gynecology, and neurology settings was recognized. The aim of this review is to update Lowe et al's ${ }^{1}$ review regarding extension of scope of physiotherapy activities in orthopedic settings.

\section{Material and methods Research questions}

The research questions for this review were:

- How is ESP practiced in orthopedic outpatients?

- What training and supervision is reported for ESP physiotherapists in orthopedic outpatients?

- What is the evidence of effectiveness of ESP physiotherapy roles in orthopedics in terms of processes, cost containment, and health outcomes?

- How does the literature identified in this review build on the findings of the Lowe et al ${ }^{1}$ review in terms of ESP physiotherapists working in orthopedic outpatients?

\section{Search strategy}

Inclusion/exclusion criteria

Types of studies

All study designs were eligible for inclusion. Literature had to be available in English, in full text, and published in peerreviewed journals.

\section{Publication dates of studies}

No start or end date was set, following the search strategy reported in Lowe et al. ${ }^{1}$

\section{Search criteria}

A PICO (participants, interventions, comparison, outcome measure) approach was used to search library databases, in which there was no defined comparison.

\section{Types of participants $(P)$}

Participants were defined as physiotherapists working in an extended scope capacity, with patients with orthopedic conditions, in non-emergency situations. If the study involved non-orthopedic conditions and the physiotherapy management was not reported separately, the study was excluded.

\section{Types of activities/interventions (I)}

Any activity or intervention provided by a physiotherapist working in an ESP capacity was reported. For the purpose of this review, extension of scope activities referred to activities which are an extension of the agreed scope of physiotherapy practice in Australia, or stated extension of physiotherapy scope of practice relevant to another country. We used the following definitions for this purpose.

\begin{abstract}
Advanced scope of practice - "A role that is within currently recognized scope of practice for that profession, but that through custom and practice has been performed by other professions. The advanced role would require additional training, competency development, as well as significant clinical experience and formal peer recognition. This role describes the depth of practice". ${ }^{2}$

Extended scope of practice - "A role that is outside the currently recognized scope of practice and requires legislative change. Extended scope of practice requires some method of credentialing following additional training, competency development, and significant clinical experience. Examples include prescribing, injecting, and surgery. This role describes the breadth of practice". ${ }^{2}$
\end{abstract}

Types of comparison interventions (C)

There was no defined comparison intervention.

\section{Types of outcome measures $(\mathrm{O})$}

Any process, cost, or health outcome was included. Such measures may include, but are not limited to, waiting times, referral rates, suitability of referrals, percentage of patients who were managed entirely by the ESP physiotherapist, and diagnostic ability of the ESP physiotherapists.

\section{Search methods for identification of studies}

All available electronic library databases were searched, using a composite and comprehensive set of search terms which were modified as required, for application to specific databases (OvidSP and Ebsco Host) and Google Scholar. See Appendix 1 for details. 


\section{Data collection and analysis}

\section{Selection of studies}

Titles and abstracts of papers identified in the search were screened for relevance and duplicates removed, before full texts were viewed to determine suitability for inclusion.

\section{Hierarchy of evidence}

The hierarchy of evidence of each included study was determined according to the National Health and Medical Research Council (NHMRC) Hierarchy of Evidence. ${ }^{3}$

\section{Assessment of risk of bias in included studies}

Lowe et al's ${ }^{1}$ review indicated that a range of study designs may be identified from this search. The potential for risk of bias in the included studies was determined initially by the allocation to the hierarchy of evidence. If studies of Levels I, II, or III_1 were identified, critical appraisal of methodological quality would be undertaken using purposebuilt key criteria relevant to the area (nonbiased selection of subjects, nonbiased allocation into treatment groups, and comparison with Gold Standard processes/outcomes). We assumed that the inherent methodological bias in the lower hierarchy studies was likely to distort the believability of any findings.

\section{Data extraction and management}

Extracted data was entered into a purpose-built Microsoft Excel sheet. Extracted data included the country of origin, research question, level of evidence, study design, patient types, ESP tasks, clinic type, a range of service delivery issues if and when reported (other health professionals involved in service delivery, appointment duration, methods of obtaining patients, training, protocols, supervision/accountability), and cost, health, and process outcome measures.

\section{Data analysis}

Data was analyzed descriptively, by considering the history and design of the available research, the processes by which, and the environments within which, ESP physiotherapists worked, the activities they undertook in the workplace (and how/if these activities were monitored/supervised), contexts of work, and any reported outcomes.

\section{Results \\ Description of studies}

One thousand and seventy-one studies were identified in the search. The ACT Government Health Directorate identified one additional, recently published study ${ }^{4}$ which was not yet library-indexed. This study was relevant and was included in the review. One thousand and forty-three studies were excluded as they were duplicates or were not relevant to the review, based on the title and abstract. Five studies were not published as full text, and a further twelve studies were excluded as not being relevant, upon screening the full text. One of these had been duplicate-published in two different journals, and another was not published in a peer-reviewed journal. The reasons for excluding the other studies are included in the consort diagram (Figure 1). Twelve studies were therefore included in the study.

\section{Study designs and levels of evidence}

Table 1 reports the author, country, type of research question, the hierarchy of evidence, and the design of the study. Two types of research questions were reported, evaluation and diagnostic, with the highest hierarchy found in the diagnostic studies (two Level II studies). The most common evaluation designs were Levels III_2 and III_3. The studies in italics were found in the 2008 review, the normal font studies were those which had been published subsequently.

The literature base, which consisted of two Level III_2 intervention studies, ${ }^{4,5}$ six lower (III_3 and IV) hierarchy intervention studies, ${ }^{6-11}$ and four diagnostic, ${ }^{12-15}$ is reported in Table 1. Two of the diagnostic studies ${ }^{13,14}$ had the highest level of hierarchy of literature identified in the search.

Morris et al ${ }^{4}$ investigated the effectiveness of physiotherapists working in a telephone triage role, which is considered within scope. However one of the management options for patients in this study was attending a multidisciplinary clinic in which an ESP physiotherapist was being trained and supervised. The extracted data for this review reports only on the ESP management component of this study.

Included studies were published from 1999 to 2011, as indicated in Figure 2. There has been no clear improvement in the bias inherent in study designs used or the types of studies published (eg, intervention, diagnostic), over this time period.

\section{Critical appraisal}

The relevant critical appraisal scores are reported in Table 2. Only two studies ${ }^{12,13}$ were eligible for critical appraisal. Both were diagnostic studies which used non-biased selection of subjects, however only Gardiner and Turner ${ }^{13}$ made comparisons with a Gold Standard. 


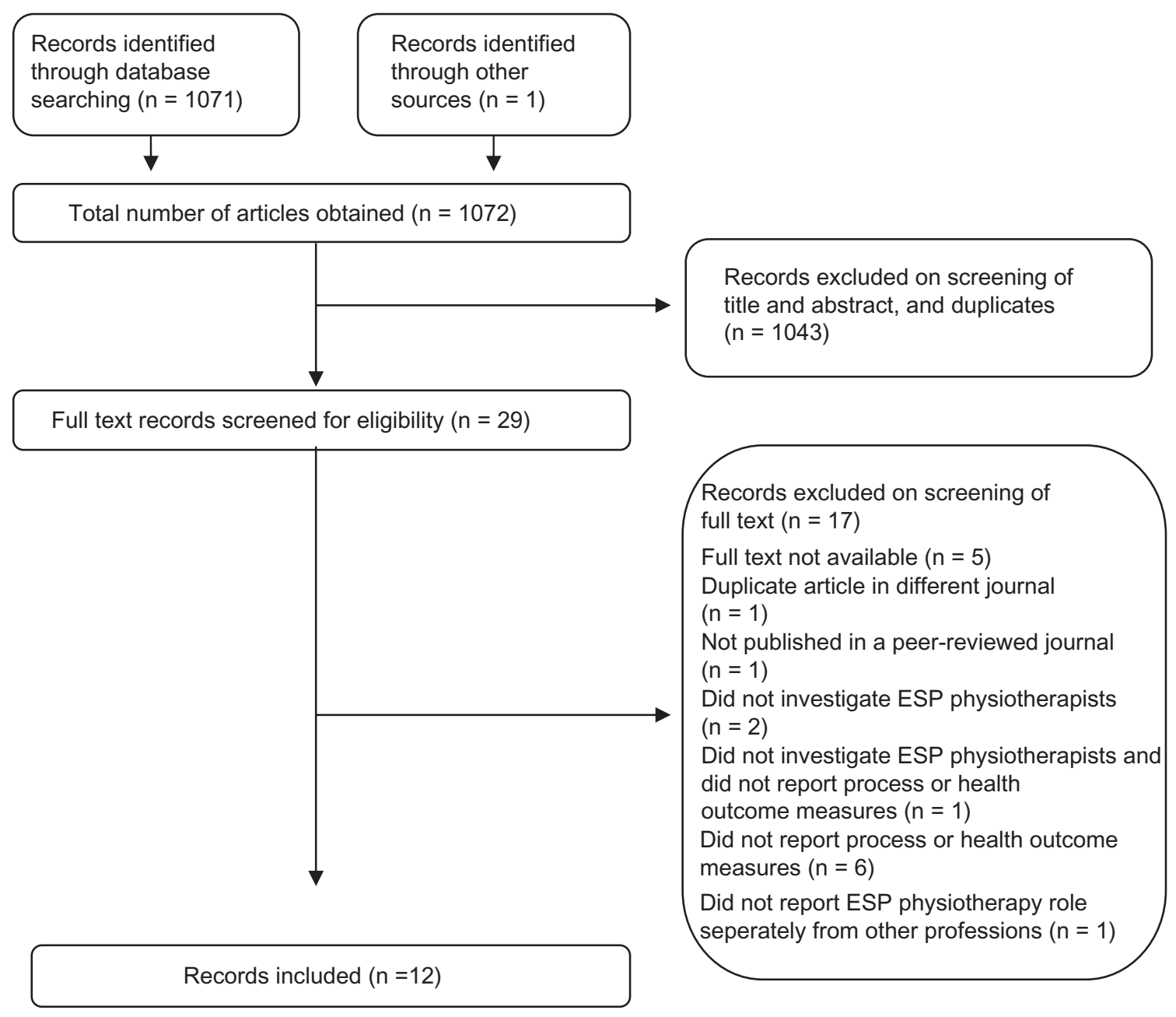

Figure I Consort diagram.

Abbreviation: ESP, extended scope practice.

\section{Tasks}

ESP physiotherapy management tasks were variously reported as injection therapy, ${ }^{5,713}$ or removal of plaster of Paris and k-wires. ${ }^{10}$ ESP physiotherapists were permitted to request further investigations such as radiographs, $5,7,8,10,11,13$ and blood tests. ${ }^{8,11,13}$ Referrals could be made by ESP physiotherapists to other allied health professionals, ${ }^{5,13,15}$ or medical professionals. ${ }^{5-8,11,13-15}$ More specifically, the medical referrals could be to orthopedics, ${ }^{5-8,11,14}$ rheumatology, ${ }^{8}$ or pain clinics. ${ }^{8}$ In some cases, the ESP physiotherapist may list patients for surgery. ${ }^{13,15}$

ESP physiotherapists did not universally have complete autonomy in performing these tasks. Some had to have X-ray requests 'signed off' by a medical consultant ${ }^{10}$ and others had to discuss referrals, surgery listings, and requests for radiological investigations with the medical consultant, although the medical consultant was not required to assess the patient. ${ }^{13}$ Harrison et $\mathrm{al}^{7}$ reported that difficult cases could be discussed with medical practitioners in the orthopedic clinic, should the ESP physiotherapist require additional support.

Protocols were developed in some cases to assist the ESP physiotherapist to conduct the extension of scope tasks. ${ }^{6,810}$ These included protocols for the first physiotherapy appointment, ${ }^{6}$ examination and assessment, and for requesting further investigations and managing fractures. ${ }^{8}$ These were developed by physiotherapy and orthopedic staff, ${ }^{6}$ or ESP physiotherapists and nurse practitioners ${ }^{10}$ and were based on the orthopedic consultants' examination, assessment, and management processes. ${ }^{8,10}$

\section{Multidisciplinary approach}

The included studies highlighted the multidisciplinary mechanisms of activities in which ESP physiotherapists engaged. They were reported to work alongside other physiotherapists $^{5,12}$ and orthopedic staff including consultants, and various specialist registrars. ${ }^{7}, 13$ 
Table I Country, type of research question, level of evidence, and study design

\begin{tabular}{|c|c|c|c|c|}
\hline Study & Country & Type of research question & Level of evidence & Study design \\
\hline Blackburn et $\mathrm{al}^{6}$ & Australia & Evaluation & III_3 & Observational: retrospective cohort \\
\hline Dickens et $a l^{12}$ & UK & Diagnostic & II & $\begin{array}{l}\text { Observational: prospective cohort/agreement } \\
\text { (between disciplines) }\end{array}$ \\
\hline Gardiner and Turner ${ }^{13}$ & UK & Diagnostic & II & $\begin{array}{l}\text { Observational: retrospective audit accuracy } \\
\text { (disciplines with surgical findings) }\end{array}$ \\
\hline Harrison et al ${ }^{7}$ & UK & Evaluation & III_3 & Observational: retrospective audit \\
\hline Hattam $^{14}$ & UK & Diagnostic & III_2 & $\begin{array}{l}\text { Observational: validation of triage decision } \\
\text { with case notes }\end{array}$ \\
\hline Hattam and Smetham ${ }^{5}$ & UK & Intervention & III_2 & Intervention: quasi-experimental \\
\hline Heywood $^{8}$ & UK & Evaluation & III_3 & Observational: retrospective cohort \\
\hline Maddison et al ${ }^{9}$ & UK & Intervention & III_3 & Observational: over time comparisons \\
\hline Moloney et al ${ }^{10}$ & Ireland & Intervention & IV & Position paper about training and measurement \\
\hline Morris et $\mathrm{al}^{4}$ & Australia & Intervention & III_2 & Intervention: quasi-experimental \\
\hline Oakes $^{15}$ & UK & Diagnostic & III_2 & $\begin{array}{l}\text { Observational: retrospective validation of triage } \\
\text { decisions using case notes }\end{array}$ \\
\hline Rabey"I & UK & Evaluation & III_3 & Observational: retrospective cohort/descriptive \\
\hline
\end{tabular}

Note: Studies in italics were found in the 2008 review; the normal font studies were those which had been published subsequently.

\section{Patient referrals}

In most cases, patients were referred to an orthopedic clinic by a medical doctor (usually a general practitioner [GP]). ${ }^{6,7,-13}$ In some cases the referrals were screened by the orthopedic surgeon ${ }^{6,7,10}$ or by the ESP physiotherapist. ${ }^{9,11}$ Direct GP referrals were reported by Hattam and Smetham, ${ }^{5}$ while in a military setting, referrals were accepted from senior physiotherapists (military and civilian), medical officers, and civilian medical practitioners. ${ }^{8}$

A range of patients were seen by ESP physiotherapists, as reported in Table 3.

\section{Training}

The ESP physiotherapists had a variety of training, and years of experience. Consequently, it is difficult to recommend a standard approach for identifying suitable candidates. For instance, Gardiner and Turner ${ }^{13}$ stated that the ESP physiotherapists had additional training in requesting

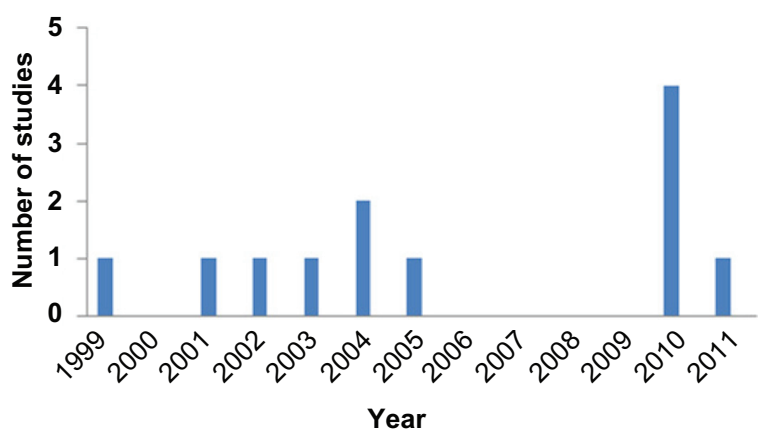

Figure 2 Graph of the number of included studies per year. investigations and performing injection therapy, although no details regarding the training were reported. In addition, while Dickens et a ${ }^{12}$ stated that the ESP physiotherapists had, on average, 5 years of experience as a senior orthopedic physiotherapist, Hattam and Smetham ${ }^{5}$ reported that their ESP physiotherapists had extensive postgraduate training, including a Diploma in Injection Therapy, over 10 years of experience working in musculoskeletal physiotherapy, and had attained a fellowship of the Society of Orthopedic Medicine. The physiotherapists in the study conducted by Blackburn et $\mathrm{al}^{6}$ had a minimum of a Masters in Musculoskeletal Physiotherapy, in addition to at least 12 years of experience in the musculoskeletal area. It is unclear in most instances whether the training reported in these studies was required prior to taking on the ESP role or whether it was provided to describe the physiotherapists who worked in this area.

Moloney et $\mathrm{a}^{10}$ examined aspects of implementation of a clinical specialist physiotherapist role, including their training, in a fracture clinic over a 6-month period. The initial 2 months was spent entirely in training. This continued over the next 4 months whilst patients were being treated. Training in the first 2 months included shadowing orthopedic consultants and registrars, other ESPs, and plaster technicians in fracture clinics. In addition, the physiotherapists attended a recognized $\mathrm{X}$-ray interpretation course, in-house courses including Radiation Protection Regulations, and they had ongoing access to study days, special interest groups, and ESP conferences. They had scheduled time to draft protocols and guidelines, and review medico-legal issues. During the third month, the physiotherapist continued to 
Table 2 Critical appraisal

\begin{tabular}{|c|c|c|c|}
\hline Study & $\begin{array}{l}\text { Non-biased selection } \\
\text { of subjects }\end{array}$ & $\begin{array}{l}\text { Non-biased allocation into } \\
\text { treatment groups }\end{array}$ & $\begin{array}{l}\text { Comparison with Gold Standard } \\
\text { processes/outcomes }\end{array}$ \\
\hline Dickens et al ${ }^{\prime 2}$ & Yes & $\begin{array}{l}\text { NA (no treatment groups, all patients } \\
\text { seen by two physiotherapists and consultant) }\end{array}$ & $\begin{array}{l}\text { No (decisions of each health provider were } \\
\text { recorded, and the surgeon had final say) }\end{array}$ \\
\hline Gardiner and Turner ${ }^{13}$ & Yes & NA (no treatment groups) & Yes (arthroscopy) \\
\hline
\end{tabular}

Note: Studies in italics were found in the 2008 review.

shadow consultants and registrars, and had a limited number of patients to review, from each of the permanent orthopedic consultants, in addition to having discussions with the consultant about patient management. Shadowing and management discussions continued in the final 3 months, in addition to the consultant assessing competency via the physiotherapist's notes, as well as the ESP writing-up selected case histories for review.

\section{Evidence to support the effectiveness of ESP physiotherapists}

The high hierarchy studies reported positive results regarding the effectiveness and efficiency of management provided by the ESP physiotherapist. The outcome measures varied between studies.

Table 3 Patient types

\begin{tabular}{|c|c|}
\hline Study & Patient types \\
\hline Blackburn et $\mathrm{al}^{6}$ & $\begin{array}{l}\text { Low back pain (including discogenic, } \\
\text { degenerative disease, spinal canal stenosis, } \\
\text { spondylolisthesis) }\end{array}$ \\
\hline Dickens et $a l^{\prime 2}$ & $\begin{array}{l}\text { Knee conditions excluding confirmed } \\
\text { fracture, previous knee surgery, } \\
\text { severe osteoarthritis, or referral } \\
\text { from another consultant }\end{array}$ \\
\hline Gardiner and Turner's & Internal derangement of the knee \\
\hline Harrison et al ${ }^{7}$ & $\begin{array}{l}\text { Shoulder problems (including } \\
\text { impingement, rotator cuff tear, } \\
\text { osteoarthritis, and adhesive capsulitis) }\end{array}$ \\
\hline Hattam $^{14}$ & Knee, shoulder, lumbar, and other sites \\
\hline Hattam and Smetham ${ }^{5}$ & Lower limb, upper limb, and spinal \\
\hline Heywood ${ }^{8}$ & Spinal \\
\hline Maddison et al ${ }^{9}$ & $\begin{array}{l}\text { Acute and chronic back pain, soft tissue } \\
\text { injury, osteoarthritis, arthritis, connective } \\
\text { tissue disease }\end{array}$ \\
\hline Moloney et al $^{10}$ & Uncomplicated fractures \\
\hline Morris et $\mathrm{al}^{4}$ & Orthopedic patients \\
\hline Oakes $^{15}$ & $\begin{array}{l}\text { Shoulder problems (impingements, muscle } \\
\text { tears and imbalance, adhesive capsulitis, } \\
\text { fractured clavicle, glenohumeral joint } \\
\text { osteoarthritis and stability, and spinal } \\
\text { referred) }\end{array}$ \\
\hline Rabey'I & Knee and lumbar spine disorders \\
\hline
\end{tabular}

Note: Studies in italics were found in the 2008 review; the normal font studies were those which had been published subsequently.
Hattam $^{14}$ reported on the nature of referrals made by ESP physiotherapists. He reported that $16.5 \%$ referrals were for investigations, $27.6 \%$ were for further investigation, and $55.9 \%$ were for surgery. Seventy-four percent of patients referred for surgery were deemed suitable for a surgical referral, $79 \%$ had an operable lesion, and $69.5 \%$ accuracy reported in determining whether surgery was required. Overall, $70.6 \%$ of referrals to consultants were considered appropriate.

\section{Diagnostic accuracy}

Two high-hierarchy diagnostic studies compared the ESP physiotherapist's diagnosis with consultants' or surgical findings. Dickens et al ${ }^{12}$ investigated the diagnosis made by a consultant and two ESP physiotherapists, on the basis of a non-invasive assessment. Following this, participants were referred for conservative treatment or arthroscopy, based upon discussion between the three professionals, in which the consultant had the final say. The accuracy of this diagnosis was determined by comparing it to arthroscopy results. If the patient improved with conservative treatment, the consultant's decision was considered to be correct, regardless of the views of the ESP physiotherapists. They found that the consultant's initial diagnosis was correct for $92 \%$ of patients, while $84 \%$ and $80 \%$ agreement was reported for the two ESP physiotherapists involved in the study, respectively.

Gardiner and Turner ${ }^{13}$ compared the orthopedic team, doctors, and ESP physiotherapist's diagnoses with arthroscopy findings $(n=128)$. The ESP physiotherapist's diagnosis agreed with the arthroscopy findings for $52 \%$ of patients, compared with $40 \%$ and $37 \%$ agreement for the orthopedic team and doctors, respectively. Furthermore, the ESP physiotherapist identified that the patient had an operable condition $100 \%$ of the time, in comparison with $79 \%$ achieved by doctors.

\section{Waiting times}

Only two high-hierarchy studies reported waiting times associated with implementing an ESP physiotherapy role. Hattam and Smetham ${ }^{5}$ reported that the average waiting 
time to see the ESP physiotherapist was 32 working days (range 4-87 days), compared with the orthopedic waiting times which were over 11 months. However, the quasiexperimental design of this study attenuates the believability of the finding.

Morris et $\mathrm{al}^{4}$ triaged some orthopedic patients to a multidisciplinary clinic, which included an orthopedic surgeon, a rheumatologist, and an ESP physiotherapist. Prior to the implementation of this service, the median time on the waiting list for an orthopedic appointment was 25.1 months (IQR: 24.3-31.3) compared with the time waiting for a triage telephone call, followed by some form of management (6.6 months [IQR: 5.8-7.5 months]). This represented a significant decrease in waiting time.

\section{Costs}

Harrison et $\mathrm{al}^{7}$ in the UK compared the cost of an orthopedic appointment with an ESP physiotherapy appointment. The ESP physiotherapy appointment was cheaper at $£ 11$ (compared with $£ 16$ for an orthopedic appointment).

Morris et al, ${ }^{4}$ in an Australian study, reported the cost of an ESP physiotherapist as AU\$58.03/hour compared with a salaried specialist (AU\$128.76/hour). The average cost of an appointment at the multidisciplinary clinic was AU\$52.59. Via this clinic, 21 patients were discharged prior to having an orthopedic appointment resulting in a decrease in costs of approximately $48 \%$.

\section{Health outcomes}

Health outcomes were rarely reported. While Harrison et $\mathrm{al}^{7}$ reported that Constant-Murley shoulder scores (which evaluate function, range of motion and pain) improved on average by 40 points, and that no patient had a decrease in their score, no comparison was made with other forms of management. Dickens et al ${ }^{12}$ reported that all patients who received conservative physiotherapy management improved. The focus of this study was determining agreement between the diagnosis made by an orthopedic consultant and ESP physiotherapist. Hence, this management was unlikely to involve any ESP techniques.

\section{Discussion}

Only one additional study ${ }^{8}$ published within the dates of the Lowe et $\mathrm{al}^{1}$ review, was included in this review. Since 2008, only six more studies have been published which reported processes and/or outcome measures related to physiotherapists working in ESP roles in orthopedic settings. However, the low levels of evidence and poor methodological quality of these studies mean that the evidence base regarding the effectiveness of physiotherapists working in extension of scope practice in orthopedics has not changed since Lowe et al's ${ }^{1}$ review. Consequently, no conclusions can be drawn regarding the effectiveness of the management of orthopedic patients by physiotherapists working in extended scope roles. The studies provide some evidence that the introduction of such roles may decrease waiting times and costs, and that some patients who had been referred to the orthopedic clinic may not require further medical management following management by an ESP physiotherapist. Perhaps the most important aspect of this review was the finding that ESP physiotherapists' ability to diagnose some musculoskeletal conditions, and accurately identify patients requiring surgery, was comparable to that of orthopedic (medical) consultants.

What constitutes extended scope of orthopedic practice for physiotherapists remains unclear. This is possibly due to the difference in traditional physiotherapy roles in different countries and even different work settings. This, therefore, constrains the differentiation between traditional physiotherapy skills (within scope), advanced physiotherapy skills (at the margins of scope), and extended scope of practice. Due to the large number of studies from the UK, the information presented is most relevant to ESP physiotherapy roles in that country. Although this may give an indication of the benefits of developing such roles elsewhere, research specific to the health service delivery processes in each country will need to be conducted to ensure that the same benefits are obtained elsewhere. This is corroborated by a recent commentary paper ${ }^{16}$ which highlights the need for establishing a business case prior to introducing ESP physiotherapy intervention, to ensure that significant impact on service delivery will occur. Furthermore, all studies were conducted in specific hospital settings, sometimes with only one ESP physiotherapist involved, which limits the generalizability and clinical utility of the results. Each ESP physiotherapist was likely to have different work experiences, different training both within the hospital and more formal settings, different protocols and varying levels of support, and contact with others in the orthopedic team.

There are persistent evidence gaps to support the widespread implementation of ESP physiotherapy roles. The positive results reported in some studies, however, indicate that further research would be of benefit. This research should aim to use high quality study designs, and investigate the ESP physiotherapy roles on a large scale, potentially over a variety of sites, and different physiotherapists. The training provided 
to ESP physiotherapists, the decision-making protocols they use, and support of others within the multidisciplinary team should be clearly reported so that the reproducibility of the health service delivery processes can be replicated elsewhere, should positive results be obtained. While process measures such as waiting times form the majority of the reported evidence, cost and health outcomes should also be considered in future studies to demonstrate that management provided by ESP physiotherapists is cost effective, beneficial, and safe for patients.

\section{Conclusion}

This review found that research into ESP physiotherapy roles in orthopedics has not considerably improved in quality or volume since the last systematic review ${ }^{1}$ was conducted in 2008. There is still no clarity on definitions of ESP roles (sufficient to distinguish them from traditional or advanced practice), and the available research continues to be of lower hierarchy, threatened by bias and sitespecific. The lack of standardized training underpinning ESP physiotherapy practice is evident, despite the country of origin of the research, and there are large differences in autonomy in decision-making. The literature suggests that ESP physiotherapists may be comparable with medical doctors in terms of clinical decision-making pertaining to patients with orthopedic conditions, and there are indications that ESP physiotherapy services may improve the efficiency of outpatient management pathways for orthopedic patients.

\section{Acknowledgment}

The iCAHE team for conducting the search.

\section{Disclosure}

The authors report no conflicts of interest in this work.

\section{References}

1. Lowe J, Prior M. A Systematic Review of the Literature on extended scope of practice physiotherapy: Physiotherapy extended scope of practice project. ACT Government Health Directorate: ACT Government; 2008. Available from: http://www.health.act.gov.au/c/health? $\mathrm{a}=$ sendfile $\& \mathrm{ft}=\mathrm{p}$ \&fid=1301227758\&sid=. Accessed January 11, 2012.

2. APA. Position Statement: Scope of Practice. Australian Physiotherapy Association: Victoria; 2009. Available from: http://physiotherapy.asn. au/images/Document_Library/Position_Statements/2014\%20scope $\% 20$ of $\% 20$ practice.pdf. Accessed November 21, 2011.

3. NHMRC. NHMRC levels of evidence and grades for recommendations for developers of guidelines. National Health and Medical Research Council: Australia Government; 2009. Available from: http://www. nhmrc.gov.au/_files_nhmrc/file/guidelines/evidence_statement_form. pdf. Accessed January 23, 2012.

4. Morris J, Grimmer-Somers K, Kumar S, et al. Effectiveness of a physiotherapy-initiated telephone triage of orthopedic waitlist patients. Patient Related Outcome Measures. 2011;2:151-159.

5. Hattam P, Smetham A. Evaluation of an orthopaedic screening service in primary care. Clin Perform Qual Health Care. 1999;7(3):121-124.

6. Blackburn M, Cowan S, Cary B, Nall C. Physiotherapy-led triage clinic for low back pain. Aust Health Rev. 2009;33(4):663-670.

7. Harrison J, Rangan A, Shetty A, Robinson C. Reducing waiting times: physiotherapy shoulder assessment clinic. Brit J Ther Rehabil. 2001;8:57-59.

8. Heywood J. Specialist physiotherapists in orthopaedic triage-the results of a military spinal triage clinic. J R Army Med Corps. 2005;151(3):152-156.

9. Maddison P, Jones J, Breslin A, et al. Improved access and targeting of musculoskeletal services in northwest wales: targeted early access to musculoskeletal services (teams) programme. BMJ. 2004;329(7478):1325-1327.

10. Moloney A, Dolan M, Shinnick L, Murphy M, Wallace L. A 6-month evaluation of a clinical specialist physiotherapist's role in a fracture clinic. Physiother Ireland. 2009;30(1):8-15.

11. Rabey M. Orthopaedic physiotherapy practitioners: surgical and radiological referral rates. Clinical Governance. 2009;14(1):15-19.

12. Dickens V, Ali F, Gent H, Rees A. Assessment and diagnosis of knee injuries: the value of an experienced physiotherapist. Physiother. 2003;89:417-422.

13. Gardiner J, Turner P. Accuracy of clinical diagnosis of internal derangement of the knee by extended scope physiotherapists and orthopaedic doctors: retrospective audit. Physiother. 2002;88:153-157.

14. Hattam P. The effectiveness of orthopaedic triage by extended scope physiotherapists. Clinical Governance. 2004;9:244-252.

15. Oakes H. Orthopaedic shoulder clinic diagnosis and treatment plan audit. Clinical Governance. 2009;14(2):126-133.

16. Gilmore LG, Morris JH, Murphy K, Grimmer-Somers K, Kumar S. Skills escalator in allied health: a time for reflection and refocus. $J$ Healthcare Leadership. 2011;3:53-58. 
Appendix I Scoping search for physiotherapists ESP in Orthopedics and Emergency Department

\begin{tabular}{|c|c|}
\hline Databases & Search terms \\
\hline Google scholar & Physiotherapy + ESP + ortho \\
\hline EBSCO Host & Physiotherap* OR "physical therap*” \\
\hline Academic Search Premier, AgeLine, CINAHL, eBook Collection & AND \\
\hline (EBSCOhost), Education Research Complete, E-Journals, ERIC, & "Extended scope" OR "extended practice" OR ESP \\
\hline Health Business Elite, Health Source-Consumer Edition, & AND \\
\hline \multirow[t]{16}{*}{ Health Source: Nursing/Academic Edition, SPORTDiscus } & Orthopaedic* OR orthopedic* \\
\hline & Physiotherap* OR "physical therap*” \\
\hline & AND \\
\hline & "Extended scope" OR "extended practice" OR ESP \\
\hline & AND \\
\hline & Emergency OR urgent \\
\hline & Physiotherap* OR "physical therap*” \\
\hline & AND \\
\hline & "Extended scope" OR "extended practice" OR ESP \\
\hline & AND \\
\hline & Bone OR muscle \\
\hline & physiotherap* OR "physical therap*” \\
\hline & AND \\
\hline & "Extended scope" OR "extended practice" OR ESP \\
\hline & AND \\
\hline & Musculoskelet* \\
\hline \multirow[t]{20}{*}{ OvidSP } & Physiotherap* OR “physical therap*” \\
\hline & AND \\
\hline & "Extended scope" OR "extended practice" OR ESP \\
\hline & AND \\
\hline & Orthopaedic* OR orthopedic* \\
\hline & Physiotherap* OR "physical therap*" \\
\hline & AND \\
\hline & "Extended scope" OR "extended practice" OR ESP \\
\hline & AND \\
\hline & Emergency OR urgent \\
\hline & Physiotherap* OR "physical therap*" \\
\hline & AND \\
\hline & "Extended scope" OR "extended practice" OR ESP \\
\hline & AND \\
\hline & Bone OR muscle \\
\hline & Physiotherap* OR "physical therap*” \\
\hline & AND \\
\hline & "Extended scope" OR "extended practice" OR ESP \\
\hline & AND \\
\hline & Musculoskelet* \\
\hline
\end{tabular}

Abbreviation: ESP, extended scope practice.

Journal of Multidisciplinary Healthcare

\section{Publish your work in this journal}

The Journal of Multidisciplinary Healthcare is an international, peerreviewed open-access journal that aims to represent and publish research in healthcare areas delivered by practitioners of different disciplines. This includes studies and reviews conducted by multidisciplinary teams as well as research which evaluates the results or conduct of such teams or healthcare processes in general. The journal covers a wide range of areas and welcomes submission from practitioners at all levels, from all over the world. The manuscript management system is completely online and includes a very quick and fair peer-review system. Visit http://www.dovepress.com/testimonials.php to read real quotes from published authors.

\footnotetext{
Submit your manuscript here: http://www.dovepress.com/journal-of-multidisciplinary-healthcare-journal
} 